\title{
The Lung Function Impairment in Non-Atopic Patients With Chronic Rhinosinusitis and Its Correlation Analysis
}

\author{
Linghao Zhang ${ }^{1}$ Lu Zhang ${ }^{1} \cdot$ Chun-Hong Zhang ${ }^{1}$ Xiao-Bi Fang ${ }^{1} \cdot$ Zhen-Xiao Huang $^{2} \cdot$ Qing -Yuan Shi ${ }^{1}$ Li-Ping Wu ${ }^{1}$ \\ Peng Wu ${ }^{1} \cdot$ Zhen-Zhen Wang $^{1} \cdot$ Zhi-Su Liao $^{1}$ \\ ${ }^{1}$ Department of Otolaryngology, First Affiliated Hospital of Wenzhou Medical University, Wenzhou; ${ }^{2}$ Department of Otolaryngology-Head and \\ Neck Surgery, Beijing Tongren Hospital, Capital Medical University, Ministry of Education, Beijing, China
}

Objectives. Chronic rhinosinusitis (CRS) is common disease in otorhinolaryngology and will lead to lower airway abnormality. However, the only lung function in CRS patients and associated factors have not been much studied.

Methods. One hundred patients with CRS with nasal polyps (CRSwNP group), 40 patients with CRS without nasal polyps (CRSsNP group), and 100 patients without CRS were enrolled. The difference in lung function was compared. Meanwhile, CRSwNP and CRSsNP group were required to undergo a bronchial provocation or dilation test. Additionally, subjective and objective outcomes were measured by the visual analogue scale (VAS), 20-item Sino-Nasal Outcome Test (SNOT-20), Lund-Mackay score, Lund-Kennedy endoscopic score. The correlation and regression methods were used to analyze the relationship between their lung function and the above parameters.

Results. The forced expiratory volume in 1 second $\left(\mathrm{FEV}_{1}\right)$ and forced expiratory flow between $25 \%$ and $75 \%$ of forced vital capacity $\left(\mathrm{FEF}_{25-75}\right)$ of $\mathrm{CRSwNP}$ group were significantly lower than other groups $(P<0.05)$. On peak expiratory flow, there was no difference between three groups. In CRSwNP group, $\mathrm{FEV}_{1}$ was negatively correlated with peripheral blood eosinophil count (PBEC) and duration of disease $(r=-0.348, P=0.013$ and $r=-0.344, P=0.014$, respectively), $\mathrm{FEF}_{25-75}$ negatively with VAS, SNOT-20 ( $r=-0.490, P=0.028$ and $r=-0.478, P=0.033$, respectively) in CRSsNP group. The incidence of positive bronchial provocation and dilation test was lower in CRSwNP group (10\% and $0 \%$, respectively), with both $0 \%$ in CRSsNP group. The multiple linear regression analysis indicated that change ratio of $\mathrm{FEV}_{1}$ before and after bronchial provocation or dilation test were correlated with PBEC in CRSwNP group $(\beta=0.403, P=0.006)$.

Conclusion. CRS leading to impaired maximum ventilation and small airway is associated with the existence of nasal polyp. Lung function impairments can be reflected by PBEC, duration, VAS, and SNOT-20. In CRSwNP patients, PBEC is independent predictor of $\mathrm{FEV}_{1}$ change ratio.

Keywords. Chronic Rhinosinusitis; Lung Function; Bronchial Hyperreactivity; Bronchial Provocation Tests

\section{INTRODUCTION}

- Received October 24, 2015

Revised December 8, 2015

Accepted January 9, 2016

- Corresponding author: Zhi-Su Liao

Department of Otolaryngology, First Affiliated Hospital of Wenzhou Medical

University, Nan Bai Xiang Street, Ouhai District,Wenzhou 325000, China

Tel: +86-574-55579175, Fax: +86-577-85221608

E-mail: zlh268401@163.com
Chronic rhinosinusitis (CRS) is a group of diseases with two or more symptoms persisting for more than 12 weeks. Symptoms include facial pain/pressure, purulent nasal discharge, nasal obstruction, and decreased sense of smell in the setting of chronic inflammation confirmed through endoscopy or radiographic studies [1]. The prevalence of CRS in the general population is

Copyright (C) 2016 by Korean Society of Otorhinolaryngology-Head and Neck Surgery.

This is an open-access article distributed under the terms of the Creative Commons Attribution Non-Commercial License (http://creativecommons.org/licenses/by-nc/4.0)

which permits unrestricted non-commercial use, distribution, and reproduction in any medium, provided the original work is properly cited. 
quite high. The National Ambulatory Medical Care Survey revealed $14 \%$ to $16 \%$ of the US population suffered from CRS, which led to decreased productivity, delayed work time [2,3]. Since the guideline of "allergic rhinitis and its impact on asthma (ARIA)" [4] was presented in 2001, researches on correlation of upper and lower airway have been increasing more thorough. Studies reported a few CRS patients suffered from lower airway diseases at same time, such as asthma and small airway diseases [5]. Lots of researches demonstrated patients with upper airway diseases often had abnormal lung function of lower airway. Williamson et al. [6] found there was asymptomatic airway impairments in CRS patients with nasal polyps. Ciprandi et al. [7] reported $53.7 \%$ patients with persistent allergic rhinitis were subjected to decreased parameters of lung function. But, there were fewer researches concerning lung function in CRS patients and its correlation. Thus, the aim of this study was to determine the lung function in CRS patients and associated factors.

\section{MATERIALS AND METHODS}

\section{Patients}

This prospective study was performed on patients undergoing surgical therapy in Department of Otolaryngology, First Affiliated Hospital of Wenzhou Medical University, Wenzhou, China from March 2012 to 2015. This study was approved by the Research Ethics Committee of First Affiliated Hospital of Wenzhou Medical University, and informed consents were then received from all participants. The criteria excluded patients diagnosed with pregnant, severe heart, lung, liver, kidney, blood system disease, upper respiratory infections, pulmonary diseases like asthma and took regular medication such as hormone, antihistamine drug, and bronchodilator. Finally, those who did not complete lung function test as well as subjective and objective measurements were also excluded. Patients were classified into three groups: CRS with nasal polyps (CRSwNP group), CRS without nasal polyps (CRSsNP group), and control group.

\section{Demographic and clinical characteristics of three groups}

Demographic and clinical characteristics were recorded, including the gender, age, smoking, atopy, peripheral blood eosinophil

\section{H I G H L I G H T S}

- Impaired maximum ventilation and small airway in patients with chronic rhinosinusitis (CRS) were related with the existence of nasal polyp.

- Peripheral blood eosinophil count (PBEC), duration and subjective outcomes can reflect impaired lung function of CRS patients.

- PBEC was independent predictor of change ratio of $\mathrm{FEV}_{1}$ in CRS with nasal polyps patients. count (PBEC), X-ray of chest for three groups, and duration of disease, sinus surgery number for CRSwNP and CRSsNP group.

\section{Skin prick test}

Each patient underwent skin-prick test by intradermal prickpuncture of Dermatophagoides farinae, house-dust mites, cockroach, cat hair, dog hair, feathers, white birch, phoenix tree, cotton fibre and so on. The largest diameter of the wheal of each particular test is measured, a positive being a wheal of $\geq 3 \mathrm{~mm}$ [8]. Histamine was used as a positive control and $0.9 \%$ sodium chloride solution as negative control.

\section{Lung function test}

Conventional ventilation function test in three groups was performed using a computerized, pneumotachograph spirometer (MasterScreen Diff; Jaeger, Hoechberg, Germany). Recorded parameters were forced expiratory volume in 1 second $\left(\mathrm{FEV}_{1}\right)$, peak expiratory flow (PEF), forced expiratory flow between $25 \%$ and $75 \%$ of forced vital capacity $\left(\mathrm{FEF}_{25-75}\right)$ of predicted value.

In control group, patients were given the above test and record. However, in CRSwNP and CRSsNP group, patients were given the above same test and record, and then bronchial provocation or dilation test based on final result of $\mathrm{FEV}_{1}$ of predicted value. They were given bronchial provocation test when $\mathrm{FEV}_{1} \geq$ $80 \%$ of predicated value, bronchial dilation test when $\mathrm{FEV}_{1}$ $<80 \%$ of predicated value. In bronchial provocation test, patients were required to inhale increasing methacholine by a quantitative pulverization machine $(4 \mathrm{mg} / \mathrm{mL}, 0.06 \mathrm{mg} ; 4 \mathrm{mg} /$ $\mathrm{mL}, 0.07 \mathrm{mg} ; 32 \mathrm{mg} / \mathrm{mL}, 0.42 \mathrm{mg} ; 32 \mathrm{mg} / \mathrm{mL}, 0.55 \mathrm{mg} ; 32 \mathrm{mg} /$ $\mathrm{mL}, 0.65 \mathrm{mg} ; 32 \mathrm{mg} / \mathrm{mL}, 0.75 \mathrm{mg}$; respectively), with $0.9 \%$ sodium chloride solution as control. Ventilation function test was performed every 2 minutes. The total dose of methacholine was as high as $2.5 \mathrm{mg}$. PD20-FEV 1 (provoking dose needed when FEV1 decreased by $20 \%$ of predicated value) $\leq 2.5 \mathrm{mg}$ was considered an indication of positive bronchial provocation test. The increasing ratio of $\mathrm{FEV}_{1} \geq 15 \%$ and absolute increasing value $\geq$ $200 \mathrm{~mL}$ after inhaling $400 \mu \mathrm{mol}$ was considered an indication of positive bronchial dilation test. $\mathrm{FEV}_{1}$ of predicted value was again recorded when bronchial provocation or dilation test was completed. The change ratio of $\mathrm{FEV}_{1}$ before and after bronchial provocation or dilation test was calculated by the equation: difference of $\mathrm{FEV}_{1}$ of predicted value before and after bronchial provocation or dilation test/ $/ \mathrm{FEV}_{1}$ of predicted value before bronchial provocation or dilation test.

\section{Subjective and objective assessment of CRS}

The visual analogue scale (VAS), 20-item Sino-Nasal Outcome Test (SNOT-20), Lund-Mackay score (LMS) and Lund-Kennedy endoscopic score (LKS) was used for subjective and objective assessment of CRS. The VAS and SNOT-20 were finished by patients with CRS, LMS, and LKS by professional otolaryngologist.

The VAS was performed by CRS patients to indicate severity 
of their symptoms by choosing a segment on one line according to the subjective feeling. The possible range for each symptom was 0 to 10. "Mild" was defined as being 0 to 3 inclusive, "moderate" as $>3$ to 7 inclusive and "severe" $>7$ to 10 inclusive [9]. The SNOT-20 was used to assess health-related quality of life of CRS patients, involving 20 items of experiences that bother them in life. The possible range for each item was 0 to 5 , and the total score 0 to 100 [10].

The coronal computed tomography (CT) scans of paranasal sinus were all completed in First Affiliated Hospital of Wenzhou Medical University. The LMS was used to rank the subjective appearance of CT scan. The sinuses involved included the maxillary, anterior ethmoidal, posterior ethmoidal, sphenoidal, and frontal sinuses. The possible score for each side ranged from 0 to 12 , and the total score from 0 to 24 [11]. The LKS was used to rank the subjective appearance of the sinus endoscopy. The items for measurement included polyp, edema, discharge, scarring, and crusting. The possible scores for each side were 0 to 10 , and the total score 0 to 20 [11]. Two investigators who were blinded to the patient groups evaluated all the LKS scores.

\section{Statistical analysis}

Statistical analysis was performed using SPSS ver. 17.0 (SPSS Inc., Chicago, IL, USA). Descriptive data was presented as mean and standard deviation for parametric data, median and range for nonparametric data. Chi-square test was used to compare categorical variables, while comparisons among three groups were performed using $F$-test for normally distributed variables and Kruskal Wallis test for non-parametric variables, as required. A $P<0.05$ was considered to be significant for double-sided comparisons. Pearson or Spearman correlation coefficient was used to analyze relationship between both variables. Multiple linear regression was performed for assessing the predictor of change ratio of $\mathrm{FEV}_{1}$.

\section{RESULTS}

\section{Patients' characteristics}

Two hundred and forty patients undergoing surgical therapy inFirst Affiliated Hospital of Wenzhou Medical University during

Table 1. Demographic and clinical characteristics of three groups

\begin{tabular}{lccc}
\hline Characteristic & CRSwNP $(n=100)$ & CRSsNP $(n=40)$ & Non-CRS $(n=100)$ \\
\hline Sex (male:female) & $66: 34$ & $16: 24$ & $58: 42$ \\
Age $(\mathrm{yr})$ & $55 \pm 10$ & $51 \pm 13$ & $58 \pm 9$ \\
Smokers & $40(40)$ & $10(25)$ & $36(36)$ \\
Atopy & $10(10)$ & $2(5)$ & $6(6)$ \\
PBEC $\left(10^{9} / \mathrm{L}\right)$ & $0.28 \pm 0.43$ & $0.18 \pm 0.16$ & 0.034 \\
LMS & $15.70 \pm 5.50$ & $7.45 \pm 4.06$ & $0.21 \pm 0.17$ \\
FEV $(\%$ of predicted) & $89.72 \pm 13.07$ & $93.98 \pm 12.66$ & $1.42 \pm 1.39$ \\
PEF $(\%$ of predicted) & $89.03 \pm 17.23$ & $95.06 \pm 12.98$ & $100.07 \pm 14.12$ \\
FEF $25-75$ of predicted) & $69.85 \pm 24.54$ & $87.59 \pm 21.49$ & $91.13 \pm 17.41$ \\
\hline
\end{tabular}

Values are presented as mean \pm SD or number (\%).

CRSwNP, chronic rhinosinusitis with nasal polyps; CRSsNP, chronic rhinosinusitis without nasal polyps; CRS, chronic rhinosinusitis; PBEC, peripheral blood eosinophil count; LMS, Lund-Mackay score; FEV 1 , forced expiratory volume in 1 second; PEF, peak expiratory flow; FEF $F_{25-75}$, forced expiratory flow between $25 \%$ and $75 \%$ of forced vital capacity.
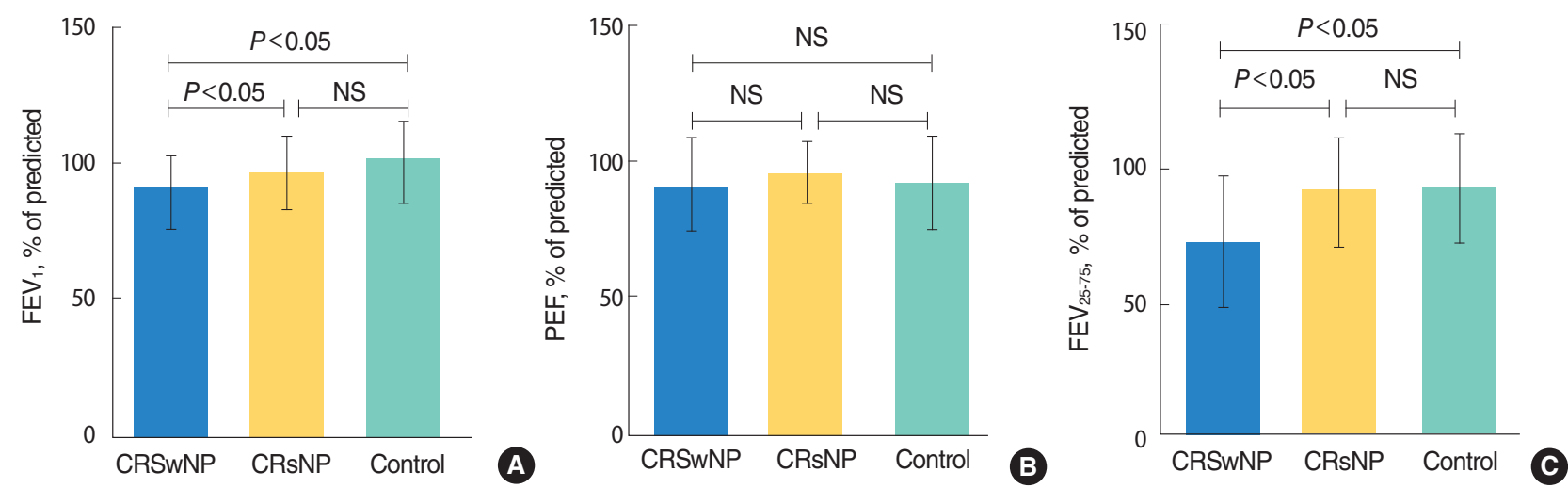

Fig. 1. Forced expiratory volume in 1 second $\left(F E V_{1}\right)$, peak expiratory flow (PEF), forced expiratory flow between $25 \%$ and $75 \%$ of forced vital capacity $\left(\mathrm{FEF}_{25-75}\right)$ in $\mathrm{CRSWNP}_{\mathrm{N}} \mathrm{CRSsNP}$ and control group. (A, C) FEV 1 and $\mathrm{FEF}_{25-75}$ were significantly lower. (B) PEF was no significantly different among three groups. CRSwNP, chronic rhinosinusitis with nasal polyps; CRSsNP, chronic rhinosinusitis without nasal polyps; NS, no significance. 
the study period were included. There was no significant difference among the three groups in terms of gender, smoking, atopy, PBEC and PEF (Table 1). Neither was there obvious inflammation presentation in X-ray of chest among three groups. However, CT score were significantly higher in CRSwNP group compared to CRSsNP and control group. $\mathrm{FEV}_{1}$ and $\mathrm{FEF}_{25-75}$ were significantly lower in CRSwNP group compared to other groups $(P<0.01)$ (Table 1, Fig. 1). None of patients with skin prick test was positive.

Correlation analysis of $\mathrm{FEV}_{1}, \mathrm{PEF}, \mathrm{FEF}_{25-75}$ in $\mathrm{CRSWNP}_{\text {group }}$ As presented in Table 2 below, $\mathrm{FEV}_{1}$ was negatively correlated with PBEC and duration of disease $(r=-0.348, P=0.013$ and $r=-0.344, P=0.014$, respectively). However, PEF and $\mathrm{FEF}_{25-75}$ was not correlated with all the parameters.

Correlation analysis of $\mathrm{FEV}_{1}, \mathrm{PEF}, \mathrm{FEF}_{25-75}$ in $\mathrm{CRSsNP}_{\text {group }}$ As presented in Table 3 below, $\mathrm{FEF}_{25-75}$ was negatively correlated with VAS, SNOT-20 $(r=-0.490, P=0.028$ and $r=-0.478, P=$ 0.033 , respectively).

\section{A multiple linear regression model for change ratio of $\mathrm{FEV}_{1}$ before and after bronchial provocation or dilation test in CRSwNP group}

A multiple linear regression analysis was constructed of change ratio of $\mathrm{FEV}_{1}$ before and after bronchial provocation or dilation test, and it was found that PBEC was independent variable predicting change ratio of $\mathrm{FEV}_{1}$ before and after bronchial provoca-

Table 2. Correlation coefficients of $\mathrm{FEV}_{1}, \mathrm{PEF}, \mathrm{FEF}_{25-75}$ in CRSwNP group

\begin{tabular}{lccccccc}
\hline Variable & PBEC & DD & SSN & VAS & SNOT-20 & LMS & LKS \\
\hline FEV 1 & $-0.348^{*}$ & $-0.344^{*}$ & -0.078 & -0.127 & -0.033 & -0.067 & -0.130 \\
PEF & 0.042 & -0.274 & 0.014 & -0.143 & -0.128 & -0.154 & -0.176 \\
FEF $_{25-75}$ & -0.149 & -0.237 & -0.056 & -0.128 & -0.180 & -0.070 & -0.135 \\
\hline
\end{tabular}

$\mathrm{FEV}_{1}$, forced expiratory volume in 1 second; PEF, peak expiratory flow; $\mathrm{FEF}_{25-75}$, forced expiratory flow between $25 \%$ and $75 \%$ of forced vital capacity; CRSwNP, chronic rhinosinusitis with nasal polyps; PBEC, peripheral blood eosinophil count; DD, duration of disease; SSN, sinus surgery number; VAS, visual analogue scale; SONT-20, 20-item Sino-Nasal Outcome Test; LMS, Lund-Mackay score; LKS, Lund-Kennedy endoscopic score. ${ }^{*} P<0.05$.

Table 3. Correlation coefficients of $\mathrm{FEV}_{1}, \mathrm{PEF}, \mathrm{FEF}_{25-75}$ in $\mathrm{CRSsNP}$ group

\begin{tabular}{lcccccrr}
\hline Variable & PBEC & DD & SSN & VAS & SNOT-20 & LMS & LKS \\
\hline FEV ${ }_{1}$ & -0.068 & 0.388 & 0.352 & -0.030 & -0.150 & -0.196 & 0.048 \\
PEF & -0.108 & -0.108 & -0.182 & -0.034 & -0.092 & 0.218 & -0.095 \\
FEF $_{25-75}$ & -0.156 & -0.010 & -0.134 & $-0.490^{*}$ & $-0.478^{*}$ & -0.103 & -0.062 \\
\hline
\end{tabular}

$\mathrm{FEV}_{1}$, forced expiratory volume in 1 second; PEF, peak expiratory flow; $\mathrm{FEF}_{25-75}$, forced expiratory flow between $25 \%$ and $75 \%$ of forced vital capacity; CRSsNP, chronic rhinosinusitis without nasal polyps; PBEC, peripheral blood eosinophil count; DD, duration of disease; SSN, sinus surgery number; VAS, visual analogue scale; SNOT-20, 20-item Sino-Nasal Outcome Test; LMS, Lund-Mackay score; LKS, Lund-Kennedy endoscopic score. ${ }^{*} P<0.05$.

Table 4. Linear regression model summary of CRSwNP group for change ratio of $\mathrm{FEV}_{1}$ before and after bronchial provocation or dilation test $\left(R^{2}=0.300\right)$

\begin{tabular}{lrccc}
\hline Variable & $\mathrm{B}$ & $\mathrm{SE}$ & $B$ & $P$-value \\
\hline Constant & -2.459 & 5.347 & & \\
Age & 0.093 & 0.069 & 0.195 & 0.184 \\
PBEC & 4.439 & 1.544 & 0.403 & $0.006^{*}$ \\
DD & 0.007 & 0.007 & 0.158 & 0.359 \\
SSN & -0.715 & 1.176 & -0.109 & 0.546 \\
VAS & 0.065 & 0.147 & 0.100 & 0.662 \\
SNOT-20 & 0.011 & 0.234 & 0.009 & 0.961 \\
LMS & 0.211 & 0.145 & 0.243 & 0.153 \\
LKS & -0.067 & 0.254 & -0.047 & 0.795 \\
\hline
\end{tabular}

CRSwNP, chronic rhinosinusitis with nasal polyps; $\mathrm{FEV}_{1}$, forced expiratory volume in 1 second; SE, standard error; PBEC, peripheral blood eosinophil count; DD, duration of disease; SSN, sinus surgery number; VAS, visual analogue scale; SNOT-20, 20-item Sino-Nasal Outcome Test; LMS, Lund-Mackay score; LKS, Lund-Kennedy endoscopic score.

${ }^{\star} P<0.05$ indicates significant correlation between the two parameters.
Table 5. Linear regression model summary of CRSsNP group for change ratio of $F E V_{1}$ before and after bronchial provocation or dilation test $\left(R^{2}=0.562\right)$

\begin{tabular}{lrccc}
\hline Variable & $\mathrm{B}$ & $\mathrm{SE}$ & $B$ & $P$-value \\
\hline Constant & -6.324 & 4.812 & & \\
Age & 0.083 & 0.047 & 0.384 & 0.106 \\
PBEC & -4.955 & 4.321 & -0.287 & 0.276 \\
DD & -1.151 & 0.014 & -0.302 & 0.319 \\
SSN & -4.366 & 1.553 & -0.155 & 0.474 \\
VAS & 0.350 & 0.232 & 1.151 & 0.159 \\
SNOT-20 & -0.368 & 0.251 & -0.949 & 0.170 \\
LMS & 0.116 & 0.307 & 0.173 & 0.712 \\
LKS & 0.689 & 0.579 & 0.381 & 0.259 \\
\hline
\end{tabular}

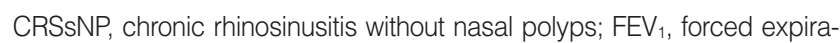
tory volume in 1 second; SE, standard error; PBEC, peripheral blood eosinophil count; DD, duration of disease; SSN, sinus surgery number; VAS, visual analogue scale; SNOT-20, 20-item Sino-Nasal Outcome Test; LMS, Lund-Mackay score; LKS, Lund-Kennedy endoscopic score.

$P<0.05$ indicates significant correlation between the two parameters. 
Table 6. Comparison of lung function parameters and PBEC

\begin{tabular}{llcccc}
\hline Group & PBEC & No. & FEV & PEF & FEF $25-75$ \\
\hline CRSwNP & Low & 78 & $92.50 \pm 12.26$ & $89.02 \pm 16.26$ & $72.33 \pm 23.67$ \\
& Moderate & 16 & $83.41 \pm 8.25$ & $96.38 \pm 16.88$ & $71.30 \pm 23.43$ \\
& High & 6 & $70.47 \pm 15.00$ & $69.53 \pm 21.66$ & $33.73 \pm 10.40$ \\
CRSsNP & P-value & & 0.004 & 0.068 & 0.028 \\
& Low & 36 & $95.16 \pm 12.78$ & $96.36 \pm 13.53$ & $89.15 \pm 20.84$ \\
& Moderate & 6 & $87.23 \pm 11.71$ & $87.67 \pm 6.20$ & $78.73 \pm 27.83$ \\
& P-value & & 0.330 & 0.297 & 0.454 \\
\hline
\end{tabular}

PBEC, peripheral blood eosinophil count; $\mathrm{FEV}_{1}$, forced expiratory volume in 1 second; PEF, peak expiratory flow; FEF $25-75$, forced expiratory flow between $25 \%$ and $75 \%$ of forced vital capacity; CRSwNP, chronic rhinosinusitis with nasal polyps; CRSsNP, chronic rhinosinusitis without nasal polyps.

tion or dilation test in CRSwNP group ( $\beta=0.403, P=0.006)$ (Table 4).

\section{A multiple linear regression model for change ratio of $\mathrm{FEV}_{1}$ before and after bronchial provocation or dilation test in CRSs- NP group}

A multiple linear regression analysis indicated that none were independent variables predicting change ratio of $\mathrm{FEV}_{1}$ before and after bronchial provocation or dilation test in CRSsNP group (Table 5).

\section{Comparison of lung function parameters and peripheral blood eosinophil count}

We defined PBEC $<0.3 \times 10^{9} / \mathrm{L}$ as low level, $0.3-0.7 \times 10^{9} / \mathrm{L}$ as moderate level and $>0.7 \times 10^{9} / \mathrm{L}$ as high level. A total of $78 \mathrm{pa}-$ tients $(78 \%)$ were classified into low level, 16 patients $(16 \%)$ were moderate level and the other six patients $(6 \%)$ were high level in $\mathrm{CRSwNP}_{\text {group. }} \mathrm{FEV}_{1}$ and $\mathrm{FEF}_{25-75}$ were significantly lower in high PBEC group compared to moderate and low level groups. However, there was no significant difference among the three groups in PEF (Table 6).

Meanwhile, $\mathrm{FEV}_{1}, \mathrm{FEF}_{25-75}$ and PEF showed no significant difference between moderate (six subjects) and low level groups (34 subjects) in CRSsNP patients. Nobody was classified into high level (Table 6).

\section{DISCUSSION}

Nowadays, there have been proposed mechanisms of association of upper and lower airway diseases as follows: (1) inflammatory secretion of nasal cavity or allergen that stimulate the lower airway; (2) nasobronchial and pharyngobronchial reflex; (3) similar histologic characteristics and mucosal susceptibility of the upper and lower airway; (4) the systemic amplification [12,13]. Normally, nasal mucosa has the function of air filtering and regulating on lower airway, and the stimulation and pathological changes of nasal mucosa affect lower airway. During CRS, swelling of nasal mucosa and retaining of secretion lead to nasal obstruction, with per nasal breathing becoming peroral breathing. Per- oral breathing aggravates bronchial paralysis induced by motion [14]. Jani and Hamilos [13] have demonstrated that the upper and lower airway disease is only one portion of systemic inflammatory response of airway, they have the common mucosal susceptibility to exogenous irritant and can be mutually affected and amplified through systemic inflammatory mediators. When inflammation occurs, eosinophil, neutrophil, and mast cell infiltration are found in the mucosa of nasal cavity, paranasal sinus and bronchus. The inflammatory mediators and cytokine released by nasal mucosa go into blood circulation, leading to systemic reaction, subsequent activation of immune cell in circulation; inflammatory cells transverse blood vessel and infiltrate into the lung, thereby results in the inflammation of lung, and then impact on lung function. Vizzaccaro et al. [15] reported that there was nasobronchial reflex between the upper and lower airway. When the mucosa of nasal cavity and paranasal sinus was stimulated, it reflexively gave rise to contraction of bronchial smooth muscle, leading to augment of intrabronchial resistance and change of lung function. The stimulation of posterior sinonasal drainage to pharynx causes bronchial contraction and decrease of airflow rate. Even in CRS patients without asthma, increased airway responsiveness was found in the exacerbation of CRS [16]. Long-term inflammatory stimulation of the bronchus causes the formation of chronic inflammation and small airway abnormality, then leading to declined pulmonary ventilation and small airway obstruction. Kariya et al. [17] found the level of interleukin-5 in nasal secretions was significantly correlated with pulmonary function in patients with CRS. These studies indicate or suggest CRS patients will suffer from impairments of lung function.

In the present study, $\mathrm{FEV}_{1}$ and $\mathrm{FEF}_{25-75}$ were significantly lower in CRSwNP patients compared to CRSsNP and non-CRS patients. However, these two parameters were no significantly different between CRSsNP and control group. $\mathrm{FEV}_{1}$ was a key parameter that reflected the maximum ventilation function, and $\mathrm{FEF}_{25-75}$ could exactly reflected the situation of airway obstruction, especially small airway abnormality. This suggested CRS could cause impairments of maximum ventilation function and small airway, which was mainly related to the existence of nasal polyps. Williamson et al. [6] also found that CRS-wNP patients 
often suffered from decreased $\mathrm{FEV}_{1}$ and $\mathrm{FEF}_{25-75}$, consistent with our results. Meanwhile, the high LMS showed a low $\mathrm{FEF}_{25}$ 75 and $\mathrm{FEV}_{1}$, meaning decreased lung function was correlated with CT scans that suggested chronic sinusitis and nasal polyp in patients without lower respiratory disease. Lee et al. [18] showed the similar result. They found CT scans suggesting chronic sinusitis and nasal polyp were associated with subclinical lower airway flow limitation even in the absence of underlying lung disease. We guessed the existence of nasal polyps would aggravate nasal obstruction and discharge of CRS patients. Serious nasal obstruction as well as postnasal drainage would enhance their stimulation of lower airway, and resulted in impaired lung function. However, its concrete mechanism remains unclear.

In CRSwNP group, $\mathrm{FEV}_{1}$ was negatively correlated with PBEC and duration of disease. It indicated that PBEC and duration of disease can reflect impairment degree of lung function of CRSwNP patients. Ciprandi et al. [19] found the disease duration was significantly associated with impairment of lung function in patients with allergic rhinitis. Patients with long duration had significantly lower $\mathrm{FEV}_{1}$ and $\mathrm{FEF}_{25-75}$ than those with short duration. In this study, PBEC and duration of CRSwNP patients aggravated impairment of lung function, which might be related with the fact that the eosinophil in filters into the lung leading to formation of inflammation and the long-term inflammatory stimulation of upper airway results in structural change of lower airway. Nevertheless, the mechanism of eosinophil in this process has not been quite clear. $\mathrm{FEF}_{25-75}$ was not correlated with all the parameters in CRSwNP group. Consistent with our results, Williamson et al.'s research [6] indicated the decrease of $\mathrm{FEF}_{25-75}$ in CRSwNP patients was not correlated with the duration and symptom score that reflects severity of CRS.

In CRSsNP group, $\mathrm{FEF}_{25-75}$ had negative correlation with VAS and SNOT-22. It showed that $\mathrm{FEF}_{25-75}$ maybe can respond to patients' subjective sensation. However, $\mathrm{FEV}_{1}$ failed to correlated with PBEC and duration of disease, indicating PBEC just can be used to evaluate lung function in CRSwNP patients.

Bronchial hyperresponsiveness (BHR) is defined as a situation in which airway is in an abnormally sensitive condition after exposure to a variety of physical, chemical and biological stimulation. BHR mostly happened in asthma, allergic rhinitis and so on. Actually, BHR was also found in CRS. BHR was measured by the bronchial provocation test. In this study, the prevalence of positive bronchial provocation test in CRSwNP and CRSsNP patients was only $10 \%$ and $0 \%$, respectively. Nevertheless, increased lower airway responsiveness could be driven by the following situations including the nasobronchial reflex in CRS, inflammatory reaction caused by smoking, per oral breathing caused by nasal obstruction, posterior sinonasal drainage of inflammatory mediator during sleep [20]. Thus, there should be quite high prevalence of positive bronchial provocation test in CRS patients. Okayama et al. [21] reported that $71 \%$ of CRS patients without allergic rhinitis and any pulmonary symptoms
(30/42), had positive methacholine bronchial provocation test. $\mathrm{Gu}$ et al. [22] found there were 19 cases of positive bronchial provocation test in $56 \mathrm{CRSwNP}$ patients, with the total positive ratio reaching $33.93 \%$. The prevalence of positive bronchial provocation test was lower in this study compared to the above studies. It may be associated with the reasons below. Firstly, the inhalation dosage of methacholine and standard for positive bronchial provocation test in this study were different from those in Okayama et al.'s study [21]; Secondly, there were a lot of CRSsNP patients (40/140) in this study, and the situations of nasal polyps were not too severe even in CRSwNP patients; Finally, there were few patients with allergy in CRSwNP and CRSsNP group, but several studies [23,24] demonstrated that allergic factors had significant effects on prevalence of BHR.

In CRSwNP group, we found decreased $\mathrm{FEV}_{1}$ and $\mathrm{FEF}_{25-75}$ was more frequently observed in patients having CRSwNP with high level of PBEC than in those having CRSwNP with moderate and low level. This indicted PBEC of CRSwNP patients can reflected decreased lung function. It showed similar result according to Tanaka et al.'s research [25]. They reported that $\mathrm{FEV}_{1} /$ FVC was significantly lower in subjects with CRSwNP than in subjects with CRSsNP, and was also lower in the group having CRSwNP with peripheral blood eosinophilia than in the group having CRSwNP without peripheral blood eosinophilia. Although the prevalence of positive bronchial provocation test in this study was lower, multiple linear regression analysis indicated that change ratio of $\mathrm{FEV}_{1}$ before and after bronchial provocation or dilation test were linearly correlated with PBEC in CRSwNP group. It suggested $\mathrm{PBEC}$ was independent predictor of change ratio of $\mathrm{FEV}_{1}$ in $\mathrm{CRSwNP}$ group $\left(R^{2}=0.300\right)$. It revealed that PBEC of CRSwNP patients reflected change ratio of $\mathrm{FEV}_{1}$. In CRSwNP patients, it was found there existed histopathological changes of chronic inflammation similar to asthma, including infiltration of eosinophil. Inflammatory reaction mediated by the eosinophil was a key factor for continuous existence of disease in many types of CRS. Therefore, the eosinophil could play an important role in variation of change ratio of $\mathrm{FEV}_{1}$ in CRS patients. Williamson et al. [6] found that serum eosinophil count was independent predictor of $\mathrm{FEF}_{25-75}$ in CRSwNP patients. Huang et al. [26] reported serum eosinophils count and LMS were independent predictors of $\mathrm{MCA}_{\mathrm{R}+\mathrm{L}}$ (bilateral minimum cross-sectional area, right and left) in CRS patients with nasal polyps and asthma. Fan et al. [27] found the prevalence of CRS had no obvious influence on change of $\mathrm{FEV}_{1}$ after inhalation of bronchial dilator in asthma patients.

This study has some limitations in the interpretation of the results. First, we excluded patients diagnosed with pulmonary diseases like Asthma, but we failed to find asthma component by provocation test in 240 candidates to exclude hidden asthmatic components. Second, differences in age between the groups can represent a bias for evaluation. Patients in CRSwNP group is higher than CRSsNP group, and differences in age may affect 
the differences in lung function.

In conclusion, our results showed that impaired maximum ventilation and small airway in patients with CRS were related with the existence of nasal polyp. PBEC, duration and subjective outcomes can reflect impaired lung function of CRS patients. Meanwhile, PBEC was independent predictor of change ratio of $\mathrm{FEV}_{1}$ in $\mathrm{CRSwNP}$ patients.

\section{CONFLICT OF INTEREST}

No potential conflict of interest relevant to this article was reported.

\section{REFERENCES}

1. Rosenfeld RM, Andes D, Bhattacharyya N, Cheung D, Eisenberg S, Ganiats TG, et al. Clinical practice guideline: adult sinusitis. Otolaryngol Head Neck Surg. 2007;137(3 Suppl):S1-31.

2. Cherry DK, Woodwell DA. National Ambulatory Medical Care Survey: 2000 summary. Adv Data. 2002;(328):1-32.

3. Anon JB, Jacobs MR, Poole MD, Ambrose PG, Benninger MS, Hadley JA, et al. Antimicrobial treatment guidelines for acute bacterial rhinosinusitis. Otolaryngol Head Neck Surg. 2004;130(1 Suppl): 1-45.

4. Bousquet J, van Cauwenberge P, Khahaev N. Allergic rhinitis and its impact on asthma. J Allergy Clin Immunol. 2001;108(5 Suppl):S 147-334.

5. Kim HY, So YK, Dhong HJ, Chung SK, Choi DC, Kwon NH, et al. Prevalence of lower airway diseases in patients with chronic rhinosinusitis. Acta Otoaryngol. 2007;127(Suppl 558):110-4.

6. Williamson PA, Vaidyanathan S, Clearie K, Barnes M, Lipworth BJ. Airway dysfunction in nasal polyposis: a spectrum of asthmatic disease? Clin Exp Allergy. 2011;41(10):1379-85.

7. Ciprandi G, Cirillo I, Vizzaccaro A, Monardo M, Tosca MA. Early bronchial airflow impairment in patients with persistent allergic rhinitis and bronchial hyperreactivity. Respir Med. 2005;99(12):160612.

8. Konstantinou GN, Bousquet PJ, Zuberbier T, Papadopoulos NG. The longest wheal diameter is the optimal measurement for the evaluation of skin prick tests. Int Arch Allergy Immunol. 2010;151(4):3435.

9. Lim M, Lew-Gor S, Darby Y, Brookes N, Scadding G, Lund VJ. The relationship between subjective assessment instruments in chronic rhinosinusitis. Rhinology. 2007;45(2):144-7.

10. Piccirillo JF, Merritt MG Jr, Richards ML. Psychometric and clinimetric validity of the 20-Item Sino-Nasal Outcome Test (SNOT-20).
Otolaryngol Head Neck Surg. 2002;126(1):41-7.

11. Lund VJ, Kennedy DW. Staging for rhinosinusitis. Otolaryngol Head Neck Surg. 1997;117(3 Pt 2):S35-40.

12. Bachert C, Vignola AM, Gevaert P, Leynaert B, Van Cauwenberge P, Bousquet J. Allergic rhinitis, rhinosinusitis, and asthma: one airway disease. Immunol Allergy Clin North Am. 2004;24(1):19-43.

13. Jani AL, Hamilos DL. Current thinking on the relationship between rhinosinusitis and asthma. J Asthma. 2005;42(1):1-7.

14. de Benedictis FM, Bush A. Rhinosinusitis and asthma: epiphenomenon or causal association? Chest. 1999;115(2):550-6.

15. Vizzaccaro A, Cirillo I, Pallestrini E, Ciprandi G. Allergic rhinitis frequently impairs bronchial function. Recenti Prog Med. 2004;95(5): 251-3.

16. McCusker CT. Use of mouse models of allergic rhinitis to study the upper and lower airway link. Curr Opin Allergy Clin Immunol. 2004; 4(1):11-6.

17. Kariya S, Okano M, Oto T, HigakiT, Makihara S, Haruna T, et al. Pulmonary function in patients with chronic rhinosinusitis and allergic rhinitis. J Laryngol Otol. 2014;128(3):255-62.

18. Lee SY, Yoon SH, Song WJ, Lee SH, Kang HR, Kim SS, et al. Influence of chronic sinusitis and nasal polyp on the lower airway of subjects without lower airway diseases. Allergy Asthma Immunol Res. 2014;6(4):310-5.

19. Ciprandi G, Cirillo I, Pistorio A. Impact of allergic rhinitis on asthma: effects on spirometric parameters. Allergy. 2008;63(3):255-60.

20. Ferguson B, Powell-Davis A. The link between upper and lower respiratory disease. Curr Opin Otolaryngol Head Neck Surg. 2003;11 (3):192-5.

21. Okayama M, Iijima H, Shimura S, Shimomura A, Ikeda K, Okayama $\mathrm{H}$, et al. Methacholine bronchial hyperresponsiveness in chronic sinusitis. Respiration. 1998;65(6):450-7.

22. Gu Xiao, Ye Ping, Chen Feng-Yi, Chen Yan-Lin, Yang Yan, Shi Li. The analysis of the characteristics of pulmonary function tests in patients with nasal polyps and clinical value. J Shandong Univ. 2011;24(10): 1-45.

23. Shaaban R, Zureik M, Soussan D, Anto JM, Heinrich J, Janson C, et al. Allergic rhinitis and onset of bronchial hyperresponsiveness: a population-based study. Am J Respir Crit Care Med. 2007;176(7): 659-66.

24. Kang SH, Kim HY, Seo JH, Kwon JW, Jung YH, Song YH, et al. Bronchial hyperresponsiveness to methacholine and AMP in children with atopic asthma. Allergy Asthma Immunol Res. 2012;4(6): 341-5.

25. Tanaka S, Hirota T, Kamijo A, Ishii H, Hatsushika K, Fujieda S, et al. Lung functions of Japanese patients with chronic rhinosinusitis who underwent endoscopic sinus surgery. Allergol Int. 2014;63(1):27-35.

26. Huang Z, Zhou B, Zhang Q, Huang Q, Sun Y,Wang M, et al.The role of upper and lower airway patency in chronic rhinosinusitis with nasal polyps and asthma. Laryngoscope. 2013;123(3):569-73.

27. Fan Y, Chen S, Qu X, Zuo K, Li X, Huang J, et al.A lower prevalence of asthma among patients with chronic rhinosinusitis in southern China. J Allergy Clin Immunol. 2011;127(2):520-2.e1-5. 